\title{
G

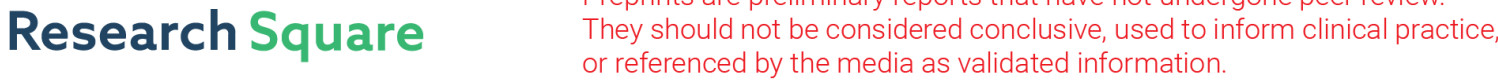 \\ Ultrasensitive Dynamic Light Scattering Immunosensing Platform for NT-ProBNP Detection Using Boronate Affinity Amplification
}

Jiaqi Hu

Nanchang University - Qianhu Campus: Nanchang University

Lu Ding

Nanchang University - Qianhu Campus: Nanchang University

Jing Chen

Nanchang University

Jinhua Fu

Jiangxi Agricultural Technology Extension Center

Kang Zhu

Nanchang University - Qianhu Campus: Nanchang University

Qian Guo

Nanchang University

Xiaolin Huang ( $\nabla$ hxl19880503@163.com )

Nanchang University - Qianhu Campus: Nanchang University https://orcid.org/0000-0002-8908-0443

Yonghua Xiong

Nanchang University

\section{Research Article}

Keywords: Dynamic light scattering, Boronate affinity, Aggregation, Immunosensor, NT-proBNP

Posted Date: October 21st, 2021

DOI: https://doi.org/10.21203/rs.3.rs-978498/v1

License: (1) (1) This work is licensed under a Creative Commons Attribution 4.0 International License.

Read Full License

Version of Record: A version of this preprint was published at Journal of Nanobiotechnology on January 6th, 2022. See the published version at https://doi.org/10.1186/s12951-021-01224-5. 


\section{Abstract}

Herein, we reported a new dynamic light scattering (DLS) immunosensing technology for the rapid and sensitive detection of glycoprotein N-terminal pro-brain natriuretic peptide (NT-proBNP). In this design, the boronate affinity recognition based on the interaction of boronic acid ligands and cis-diols was for the first time introduced to amplify the nanoparticle aggregation for enabling highly sensitive DLS transduction, thereby lowering the limit of detection (LOD) of the methodology. After covalently coupling with antibodies, magnetic nanoparticles (MNPs) were employed as the nanoprobes to selectively capture trace amount of NT-proBNP from complex samples and facilitate DLS signal transduction. Meanwhile, silica nanoparticles modified with phenylboronic acid $\left(\mathrm{SiO}_{2} @ P B A\right)$ were designed as the crosslinking agent to bridge the aggregation of MNPs in the presence of target NT-proBNP. Owing to the multivalent and fast affinity recognition between NT-proBNP containing cis-diols and $\mathrm{SiO}_{2} @ P B A$, the developed DLS immunosensor exhibited charming advantages over traditional immunoassays, including ultrahigh sensitivity with an LOD of $7.4 \mathrm{fg} \mathrm{mL}^{-1}$, fast response time (<20 min), and small sample consumption (1 $\mu \mathrm{L})$. The DLS immunosensor was further characterized with good selectivity, accuracy, precision, reproducibility, and practicability. Collectively, this work demonstrated the promising application of the designed boronate affinity amplified-DLS immunosensor for field or point-of-care testing of cis-diolcontaining molecules.

\section{Highlight}

1. We developed a new dynamic light scattering (DLS) immunosensing technology for the rapid and sensitive detection of glycoprotein NT-proBNP.

2. The boronate affinity recognition amplified nanoparticle aggregation was designed to enable highly sensitive DLS transduction.

3. The fabricated DLS immunosensor exhibited ultrahigh sensitivity with an LOD of $7.4 \mathrm{fg} \mathrm{mL}^{-1}$, fast response time (<20 min), and small sample consumption $(1 \mu \mathrm{L})$.

4. This boronate affinity amplified-DLS immunosensor has broad prospects for field or point-of-care testing of cis-diol-containing molecules.

\section{Introduction}

Heart failure (HF) is one of the most common cardiovascular diseases, which often adversely affects cardiovascular health and has become a major cause of death in humans [1]. Early diagnosis of HF contributes to timely intervention, treatment, and prognosis. N-terminal pro-brain natriuretic peptide (NTproBNP) has been considered as a clinically recognized biomarker for early diagnosis of HF [2]. In general, the population with the NT-proBNP concentrations exceeding $300 \mathrm{pg} \mathrm{mL}^{-1}$ is high risk for HF [3]. Therefore, ultrasensitive detection for NT-proBNP is crucial for accurate clinical diagnosis and prognosis 
to reduce the hospitalization and mortality rates. At present, versatile immunoassay approaches have been reported to improve the determination of NT-proBNP, including colorimetric, fluorescent, electrochemical, field effect transistor, photoelectrochemical, electrochemiluminescence, and surfaceenhanced Raman scattering [4-10]. Although current methods succeed in achieving the specific detection of NT-proBNP, they are still compromised by insufficient sensitivity, long response time, large sample consumption and limited use in the field of point-of-care $(\mathrm{POC})$ testing.

Detecting trace target analytes in highly sensitive way usually depends on the sensitive transduction techniques and the effective signal reporting strategies. In recent years, increasing interest has been focused on exploring technologies to enhance the signal transduction for designing high-performance immunosensors $[11,12]$. By coupling the strong light scattering properties of gold nanoparticles with the dynamic light scattering (DLS) technique routinely used for nanoparticle size characterization, Huo's group has pioneered the development of a DLS-based immunosensing platform for monitoring proteins [13]. Inspired by this work, many research groups have extended DLS enhanced immunosensors for the detection of small molecules, metal ions, and microorganisms [14-16]. The currently available strategies to manipulate the light scattering signals of intensity or hydrodynamic diameter $\left(D_{H}\right)$ of nanoparticles and construct DLS immunosensors mainly involve the size change of individual nanoparticles upon target binding and the target-induced nanoparticle aggregation or self-assembly upon the antigenantibody reaction [17-19]. However, owing to relatively limited signal fluctuations caused by target analytes based on antigen-antibody recognition, most of the reported DLS immunosensors have sensitivity levels of $\mathrm{ng} \mathrm{mL}^{-1}$ [20], largely confining their direct use in response to trace amounts of NT$\operatorname{proBNP}\left(\mathrm{pg} \mathrm{mL}^{-1}\right)$.

Development of strategies to amplify the nanoparticle aggregation for improving DLS signal transduction contributes to highly sensitive detection. Due to the specific interaction of boronic acid ligands with cisdiols, boronate affinity materials have attracted increasing attention in many important fields, such as disease diagnosis, cell targeting, and bacterial identification and killing [21-24]. Different from the monovalent or divalent antigen-antibody interaction, the boronate affinity reaction can readily achieve the multivalent binding between boronic acid ligands and cis-diol-containing molecules (e.g., glycoproteins), which make them act as a promising bridge to amplify the nanoparticle aggregation [25]. Leveraging this design concept, for the first time we developed a boronate affinity amplified DLS immunosensing platform for rapid and ultrasensitive detection of trace NT-proBNP, an important serum glycoprotein marker [26]. To this end, antibody-functionalized magnetic nanoparticles (MNPs) were designed as the sensitive and specific probes to selectively capture NT-proBNP from complex samples by magnetic properties and facilitate highly sensitive DLS signal transduction using light scattering properties. In addition, phenylboronic acid modified silica nanoparticles $\left(\mathrm{SiO}_{2} @ \mathrm{PBA}\right)$ with low scattering background signal were used as the crosslinking agent to amplify the MNP aggregation in the presence of target NTproBNP, which can further improve the detection performances of the methodology. By virtues of the multivalent and fast boronate affinity recognition between glycoprotein NT-proBNP and $\mathrm{SiO}_{2} @ P B A$, the developed DLS immunosensor showed the advantages of ultrahigh sensitivity $\left(7.4 \mathrm{fg} \mathrm{mL}^{-1}\right)$, rapid 
response time (<20 min), and small sample consumption $(1 \mu \mathrm{L})$. Besides, the selectivity, accuracy, precision, reproducibility, and practicability of this immunosensor were well demonstrated by an assay of NT-proBNP in human serum. Briefly, this work demonstrated the boronate affinity amplified DLS immunosensing strategy could detect NT-proBNP in rapid and highly sensitive manner, implying the feasibility for incorporating the nanoparticle crosslinking amplification strategy into DLS immunosensors to ultrasensitively monitor trace target analytes, even in field or at the POC.

\section{Materials And Methods}

\subsection{Regents and apparatus}

Carboxyl-functionalized MNPs $\left(150 \mathrm{~nm}, 25 \mathrm{mg} \cdot \mathrm{mL}^{-1}\right)$ and carboxyl-functionalized silica nanoparticles $\left(\mathrm{SiO}_{2}, 100 \mathrm{~nm}, 25 \mathrm{mg} \cdot \mathrm{mL}^{-1}\right)$ were obtained from Tianjin Baseline ChromTech Research Center (Tianjin, China). Anti-NT-proBNP monoclonal antibody (mAb) was purchased from Medix Biochemica (Espoo, Finland). Glucose, galactose, 3-aminophenylboronic acid (PBA) hydrochloride, and 1-(3-

Dimethylaminopropyl)-3-ethyl-carbodiimide (EDC) were provided from Sigma-Aldrich (St. Louis, MO, USA). Serum samples used in this study were from healthy volunteers and patients who had signed informed consent forms. All experiments using human serum samples were approved by the Medical Ethics Committee of the First Affiliated Hospital of Nanchang University. All chemicals of analytical grade were provided by Sinopharm Chemical Corp (Shanghai, China) and were used without further purification.

DLS measurements for size distribution and zeta potential were determined using a DLS nanoparticle analyzer (Malvern Nano ZSE, London, UK). were conducted on a Malvern Zetasizer Nano ZSZEN3700 DLS nanoparticle analyzer (London, UK). High-resolution transmission electron microscopy (TEM) images were obtained on a JEOL JEM 2100 microscope (Tokyo, Japan). Field-emission scanning electron microscopy (SEM) images were performed using a JEOL JSM-6701F microscope (Tokyo, Japan). Fourier transform infrared (FTIR) spectrum was measured on a Thermo Fisher Nicolet iS50 infrared spectrometer (Waltham, US). Millipore water was prepared on an Elix-3 and Milli-QA waterpurification system.

\subsection{Preparation of antibody modified MNP conjugates (MNP@mAb)}

The conjugation of MNPs and anti-proBNP mAb was conducted by using the EDC-mediated covalent coupling after electrostatic absorption. In a typical procedure, $3 \mu \mathrm{L}$ of carboxyl functionalized MNPs (20 $\mathrm{mg} \mathrm{mL}^{-1}$ ) were added into $200 \mu \mathrm{L}$ of $0.01 \mathrm{M}$ phosphate buffer (PB, pH 7.4), followed by the addition of 3 $\mu \mathrm{g}$ of anti-proBNP mAb. After gentle stirring for $30 \mathrm{~min}$ at room temperature, $1.5 \mu \mathrm{L}$ of EDC solution ( $1 \mathrm{mg}$ $\mathrm{mL}^{-1}$ ) was added into the mixed solution. After another $30 \mathrm{~min}$, the same amount of EDC was added. Finally, the resultant MNP@mAb conjugates were purified by an external magnetic field, re-dispersed in $100 \mu \mathrm{L}$ of $\mathrm{PB}(0.01 \mathrm{M}, \mathrm{pH} 7.4)$, and stored at $4^{\circ} \mathrm{C}$ until further use.

2.3 Preparation of PBA-functionalized $\mathrm{SiO}_{2}\left(\mathrm{SiO}_{2} @ \mathrm{PBA}\right)$ 
As shown in Scheme 1A, the $\mathrm{SiO}_{2} @ P B A$ conjugates were prepared through the formation of amino linkage in the presence of EDC. In brief, $200 \mu \mathrm{L}$ of $\mathrm{SiO}_{2}\left(50 \mathrm{mg} \mathrm{mL}^{-1}\right)$ was added to $2 \mathrm{~mL}$ of $0.01 \mathrm{M} \mathrm{PB}$ ( $\mathrm{pH}$ 6.0). After gentle stirring, $150 \mu \mathrm{L}$ of PBA solution $\left(50 \mathrm{mg} \mathrm{mL}^{-1}\right)$ was added, in which PBA solution was prepared by dissolving $10 \mathrm{mg}$ of 3-aminophenyl borate hydrochloride into $200 \mu \mathrm{L}$ of $0.01 \mathrm{M} \mathrm{PB}(\mathrm{pH}$ 8.0). Then, the $\mathrm{pH}$ of the mixed solution was adjusted to $6.5-7.0$ by $10 \mathrm{M} \mathrm{NaOH}$. After gentle stirring for 30 min at room temperature, $250 \mu \mathrm{g}$ of EDC was added into the mixed solution. After adding EDC three times, the mixed solution was purified by centrifugation at $12000 \mathrm{rpm}$ for $15 \mathrm{~min}$. After washed with PB $(0.01 \mathrm{M}, \mathrm{pH} 7.4)$ three times, the resulting $\mathrm{SiO}_{2} @ P B A$ products was finally obtained.

\subsection{DLS immunosensor for NT-proBNP detection}

About $6 \mu \mathrm{L}$ of MNP@mAb $\left(0.4 \mathrm{mg} \mathrm{mL}^{-1}\right)$ solution was added into $200 \mu \mathrm{L}$ of serum solution containing desired concentrations of NT-proBNP ranging from $0.01 \mathrm{pg} \mathrm{mL}^{-1}$ to $100 \mathrm{pg} \mathrm{mL}^{-1}$. After incubation at $37^{\circ} \mathrm{C}$ for $5 \mathrm{~min}$, the formed complex of MNP@mAb-NT-proBNP were collected under an external magnetic field for $10 \mathrm{~min}$ and washed three times with $0.01 \mathrm{M} \mathrm{PB}(\mathrm{pH}$ 7.4). Subsequently, the formed immunocomplex was then re-suspended in $800 \mu \mathrm{L}$ of $0.01 \mathrm{M} \mathrm{PB}$ solution (pH 7.4) containing $\mathrm{SiO}_{2} @ \mathrm{PBA}$ with the concentration of $4 \mu \mathrm{g} \mathrm{mL}^{-1}$. After incubation for $5 \mathrm{~min}$, the $D_{H}$ of the sample solution was measured on a DLS nanoparticle analyzer equipped with a green laser and an avalanche photodiode detector. The detection conditions were as follows: temperature $25^{\circ} \mathrm{C}$, detector angle $173^{\circ}$, incident laser wavelength $628 \mathrm{~nm}$, and laser power 4.0. Data were analyzed and processed using a Malvern Zetasizer Nano application software. For each sample, three DLS analysis duplicates were carried out with approximately 12 runs and each run lasting $10 \mathrm{~s}$ at a scattering angle of $173^{\circ}$. The real-world applications of the proposed DLS immunosensor was conducted by an assay of NT-proBNP in serum, in which $1 \mu \mathrm{L}$ of sample solution was added with $199 \mu \mathrm{L}$ of $0.01 \mathrm{M} \mathrm{PB}$ solution $(\mathrm{pH}$ 7.4) and then subjected to DLS analysis.

\section{Results And Discussion}

\subsection{Working principle of the developed DLS immunosensor for NT-proBNP}

Scheme 1 describes the working principle of the developed DLS immunosensor for the quantitative detection of NT-proBNP, wherein MNP@mAb was employed for magnetic enrichment of target analytes and DLS signal transduction, and $\mathrm{SiO}_{2} @ P B A$ was designed as crosslinkers to amplify the crosslinking aggregation of MNPs. When NT-proBNP was present in the sample solution, target glycoprotein was selectively captured by the MNP@mAb to form the immunocomplex of MNP@mAb-NT-proBNP. After washing to remove the supernatant, the immunocomplex was re-suspended in PB solution containing boronic acid crosslinkers, thereby inducing the MNP aggregation by the selective boronic acid ligand-cisdiol recognition between the $\mathrm{SiO}_{2} @ P B A$ and the glycoprotein. With the crosslinking aggregation of MNPs, the $D_{H}$ of the solution will remarkably increase, which can be readily measured by DLS. Specifically, when the content of NT-proBNP is more, the MNP aggregate is larger, and the $D_{H}$ of the solution is greater. By 
contrast, no obvious aggregation of MNPs was observed when the target NT-proBNP was absent, thus resulting in negligible changes in the $\mathrm{D}_{\mathrm{H}}$. Therefore, the quantitative detection of glycoproteins in unknown samples can be achieved by recording the variation in the $D_{H}$ of MNPs.

\subsection{Synthesis and characterization of MNP@mAb and SiO $2 @ P B A$}

The MNP@mAb conjugates were prepared through the formation of peptide linkage between the carboxyl group of MNPs and the amino group of anti-NT-proBNP mAb in the presence of EDC. The successful construction of the MNP@mAb was confirmed by TEM and DLS. As shown in Figure 1A, the MNPs show uniform morphology and good monodispersity before and after conjugated with mAb. DLS measurement indicates an obvious increase in the $D_{H}$ of MNPs from $139 \mathrm{~nm}$ to $149.7 \mathrm{~nm}$ after the conjugation of mAbs (Figure 1B). Figure 1C exhibits the MNP@mAb has higher zeta potential of $-36.7 \mathrm{mV}$ than that of unmodified MNPs (-56.1 mV). These results suggest the successful conjugation of MNPs with mAbs. As shown in Scheme 1A, the $\mathrm{SiO}_{2} @ P B A$ conjugates were synthesized by a similar EDC-assisted covalent coupling method. The successful modification of PBA molecules on the surface of $\mathrm{SiO}_{2}$ was verified using TEM, DLS and FTIR. Figure 1D and Figure S1 reveals that no obvious changes in the morphology and monodispersity of $\mathrm{SiO}_{2}$ were observed after modified with the PBA. Figure 1E shows that the size of $\mathrm{SiO}_{2}$ increased from $114.4 \mathrm{~nm}$ to $124 \mathrm{~nm}$ with the zeta potential reduced from $-15.0 \mathrm{mV}$ to $-31.0 \mathrm{mV}$ when PBA molecules were modified onto the surface of $\mathrm{SiO}_{2}$. Further FTIR analysis of $\mathrm{SiO}_{2} @ P B A$ was compared with $\mathrm{SiO}_{2}$. As shown in Figure 1F, FTIR spectra of $\mathrm{SiO}_{2}$ and $\mathrm{SiO}_{2} @ P B A$ present two characteristic peaks at $1057 \mathrm{~cm}^{-1}$ and $3273 \mathrm{~cm}^{-1}$, which correspond to the $\mathrm{Si}-\mathrm{O}$ band and $-\mathrm{OH}$, respectively [27]. In addition, the FTIR peaks of $\mathrm{SiO}_{2} @ P B A$ at $1633 \mathrm{~cm}^{-1}, 1541 \mathrm{~cm}^{-1}$ and $1421 \mathrm{~cm}^{-1}$ correspond to the stretching vibration of a benzene ring skeleton, the peptide bond and $-\mathrm{B}(\mathrm{OH})_{2}$, respectively, which were not observed in $\mathrm{SiO}_{2}$ alone [28]. These findings prove that $\mathrm{PBA}$ molecules were successfully modified onto the surface of $\mathrm{SiO}_{2}$.

\subsection{Confirmation of feasibility of the developed DLS immunosensor for NT-proBNP}

The feasibility of the developed DLS immunosensor for the quantitative analysis of NT-proBNP was verified by conducting a series of control experiments, including (1) MNP@mAb, (2) MNP@mAb + NTproBNP (20 pg mL $\left.{ }^{-1}\right)$, (3) MNP@mAb + SiO ${ }_{2} @ P B A$, and (4) MNP@mAb + NT-proBNP $\left(20\right.$ pg mL $\left.{ }^{-1}\right)+$ $\mathrm{SiO}_{2} @ P B A$. The formation of MNP aggreates caused by target NT-proBNP was monitored by DLS and TEM. Results in Figure 2A and Figure $\mathbf{S 1}$ present that only the co-occurrences of target NT-proBNP and $\mathrm{SiO}_{2} @ P B A$ (denoted as MNP@mAb + NT-proBNP $\left(20\right.$ pg mL $\left.\left.{ }^{-1}\right)+\mathrm{SiO}_{2} @ P B A\right)$ can result in a significant increase in the $D_{H}$ of MNP@mAb from $147 \mathrm{~nm}$ to $351 \mathrm{~nm}$. By contrast, two other groups, including MNP@mAb + NT-proBNP and MNP@mAb + SiO ${ }_{2} @ P B A$, show negligible changes in the $\mathrm{D}_{\mathrm{H}}$ compared with MNP@mAb alone. The reason for this phenomenon is that the presence of target NT-proBNP can specifically induce the MNP aggregation caused by $\mathrm{SiO}_{2} @ P B A$, which are well demonstrated by SEM and TEM imaging (Figure 2B-C). The formation of MNP aggreates caused by the $\mathrm{SiO}_{2} @ P B A$ was further 
confirmed by energy dispersive spectroscopy (EDS) mapping analysis (Figure 2D) with the coexistence of $\mathrm{Si}$ and $\mathrm{Fe}$ in the aggregates. These observations verify the feasibility of the proposed boronate affinity amplified DLS immunosensor for ultrasensitively and specifically targeting NT-proBNP.

\subsection{Optimization of experimental conditions}

To achieve the best analytical performance of the developed DLS immunosensor, several key parameters, such as the pH value, EDC amount, and labelled amount of mAbs for the preparation of MNP@mAb; the used amount of MNP@mAb for each test; the immunoreaction time of MNP@mAb and target NT-proBNP; the used amount of $\mathrm{SiO}_{2} @ P B A, p H$ value and incubation time for boronate affinity reaction between NTproBNP and $\mathrm{SiO}_{2} @ P B A$, were systematically studied. The optimized experimental conditions were evaluated by detecting the largest $D_{H}$ change using DLS. The results in Figure $3 A-C$ indicate the optimal combinations of pH value, EDC amount, and labelled amount of mAbs for the preparation of MNP@mAb were 7.5, $25 \mu \mathrm{g} \mathrm{mL}^{-1}$, and $100 \mu \mathrm{g} \mathrm{mg}^{-1}$, respectively, wherein the MNP@mAb maintains the best bioactivities for the recognition and capture of target NT-proBNP, thus giving the maximal $D_{H}$ values. Figure 3D show that the $D_{H}$ gradually increased with increasing the used amount of MNP@mAb from 1.2 $\mu \mathrm{g}$ to $2.4 \mu \mathrm{g}$ per test, and then obviously decreased with the further increase of MNP@mAb, which may be due to the presence of excess MNP@mAb to cause a reduction in the aggregate formation and size. The immunoreaction time between the MNP@mAb and the target NT-proBNP was further investigated to ensure the high sensitivity and reproducibility. Figure $3 \mathrm{E}$ shows that $5 \mathrm{~min}$ of immunoreaction time was necessary to result in the maximum $D_{H}$ with high reproducibility. In addition, to further maximize the variation in the $D_{H}$, the boronate affinity recognition between the target glycoprotein and the boracic acid crosslinker is critical to control the crosslinking aggregation of MNPs for amplifying the DLS signal and improving the sensitivity. Figure $3 \mathrm{~F}$ displays the effect of $\mathrm{pH}$ ranged from 7 to 9 on the MNP aggregation, and the results show the greatest $\mathrm{D}_{\mathrm{H}}$ value at pH 7.5. With increasing the amount of $\mathrm{SiO}_{2} @ P B A$ from $0.032 \mu \mathrm{g} \mathrm{mL}^{-1}$ to $4 \mu \mathrm{g} \mathrm{mL}^{-1}$, the $D_{H}$ value increased gradually from $224.7 \mathrm{~nm}$ to $259.6 \mathrm{~nm}$ (Figure $3 \mathrm{G}$ ). However, the $\mathrm{D}_{\mathrm{H}}$ value decreased obviously as the concentration of crosslinking agents continued to increase to $20 \mu \mathrm{g} \mathrm{mL}^{-1}$. The possible reason is that the excess crosslinker could block the remaining cisdiol sites on the glycoprotein molecules and in turn inhibit the MNP aggregation, thus giving rise to a decreased $\mathrm{D}_{\mathrm{H}}$. Figure $3 \mathrm{H}$ shows that at all NT-proBNP concentrations, the $\mathrm{D}_{\mathrm{H}}$ value reached a constant after 5 min reaction of the glycoprotein and the $\mathrm{SiO}_{2} @ P B A$, suggesting 5 min was enough to allow sensitive and reproducible DLS signal transduction for reliable quantitative analysis.

\subsection{Performance evaluation of the amplified DLS immunosensor}

The signal response of this DLS immunosensor against different concentrations of NT-proBNP was examined under the developed conditions. As shown in Figure $4 A$, the $D_{H}$ value gradually increased with the NT-proBNP concentration ranging from $0.012 \mathrm{pg} \mathrm{mL}^{-1}$ to $1100 \mathrm{pg} \mathrm{mL}^{-1}$. Figure $4 \mathrm{~B}$ shows an excellent linearity between the $D_{H}$ value and the logarithm of the NT-proBNP concentration $\left(0.012-100 \mathrm{pg} \mathrm{mL}^{-1}\right)$. The linear regression equation is described as: $y=21.885 \ln x+255.26$, with the correlation coefficient of 
0.9858. The limit of detection was $0.0074 \mathrm{pg} \mathrm{mL}^{-1}(\mathrm{~S} / \mathrm{N}=3)$, which is much lower than other previously reported immunoassays for NT-proBNP detection (Table 1), demonstrating that our immunosensor has better sensitivity. Notably, the total time to complete a test is less than 20 min, which is comparable to that of the most widely used POC device of lateral flow assay and obviously shorter than other reported immunoassays. The ultrahigh sensitivity and fast response time are ascribed to the boronate affinitybased multivalent recognition between crosslinkers and target glycoproteins and the magnetic enrichment of targets from the complex sample. Additionally, an obvious hook effect was observed at the NT-proBNP concentration of over $200 \mathrm{pg} \mathrm{mL}^{-1}$, indicating that additional sample dilution was needed to ensure accurate quantification of NT-probNP and avoid false results when the concentration of NTprobNP exceeds the hook point.

The specificity of this developed DLS immunosensor was characterized by using a series of common interfering glycoproteins, including carcinoembryonic antigen (CEA), alpha fetoprotein (AFP), human chorionic gonadotropin (HCG), and hepatitis B virus antigen (HBsAg), as well as monosaccharides such as glucose (Glc), galactose (Gal), fucose (Fuc), and nacetylneuraminic acid (Neu5Ac). Figure $4 \mathrm{C}$ indicated that only NT-proBNP can provide an apparent signal value, while other interferents show negligible changes compared with the negative control. These results indicate that the designed DLS immunosensor can specifically distinguish target glycoprotein from other interfering substances because of the specific immunorecognition between NT-proBNP and its captured antibody.

The accuracy and precision of the proposed DLS immunosensor for the quantitative detection of NTproBNP were further investigated by measuring the intra- and inter-assay recoveries. As indicated in Table S1, the intra-assay recoveries ranged from $89.8-101.13 \%$ with the coefficient of variation (CV) of 0.32$10.77 \%$, while the inter-assay recoveries varied from $80.81-104.11 \%$ with the CV of $3.91-7.87 \%$. These results indicate an acceptable accuracy and precision for quantitative determination of NT-proBNP in complex samples.

The reliability and practicability of this DLS immunosensor in actual samples were demonstrated by the detection of NT-proBNP in human serum. Forty NT-proBNP-positive serum samples collected from the First Affiliated Hospital of Nanchang University were simultaneously analyzed by the developed immunosensor and the clinical routine timed-resolved fluoroimmunoassay (TRFIA). For our method, $1 \mu \mathrm{L}$ of serum samples were diluted with $199 \mu \mathrm{L}$ of $0.01 \mathrm{M}$ PB solution ( $\mathrm{pH} 7.4)$ and then quantified, while the analysis of NT-proBNP using the TRFIA was performed according to the manufacturer's instructions. The test results obtained by these two approaches were then compared. As indicated in Table S2, among these 36 samples, 34 samples were tested with NT-proBNP as detected by our proposed DLS immunosensor method. By contrast, 30 samples were tested NT-proBNP positive by the TRFIA method. These results showed that compared with the routinely used TRFIA, the newly developed DLS immunosensor can detect lower concentrations NT-proBNP, which is attributed to its higher sensitivity (LOD, $1.48 \mathrm{pg} \mathrm{mL}^{-1}$ ) than that of the TRFIA method (70, $\mathrm{pg} \mathrm{mL}^{-1}$ ). In addition, 2 samples were simultaneously detected NT-proBNP negative by these two methods. Figure 4D and Figure S3 exhibit that the detection results of the proposed method are broadly in line with those of TRFIA with a good linear 
correlation of 0.9745 , proving the feasibility of the amplified DLS immunosensor for real-world applications in complex sample matrix.

\section{Conclusion}

In conclusion, we successfully developed an ultrasensitive immunosensor for the simple and rapid detection of NT-proBNP in complex samples by coupling boronate affinity-mediated crosslinking aggregation with DLS transduction. For this purpose, $\mathrm{SiO}_{2} @ P B A$ was designed as the crosslinking agent to control the MNP aggregation and amplify the DLS signal in the presence of target NT-proBNP, thus contributing to the detection sensitivity. Thanks to the multivalent and rapid boronate affinity reaction, this proposed DLS immunosensor has the advantages of high sensitivity $\left(7.4 \mathrm{fg} \mathrm{mL}^{-1}\right)$, short response time (20 min), and small sample consumption $(1 \mu \mathrm{L})$, exhibiting great potential for POC use. The analytical performances of this immunosensor were demonstrated in selectivity, accuracy, and practicability. Moreover, the feasibility and reliability of this approach for real-world applications was characterized by an assay of trace NT-proBNP in human serum, which was further corroborated by the clinical routine TRFIA. Briefly, the boronate affinity-amplified DLS immunosensing platform provides a promising analytical tool for POC detection of cis-diol-containing compounds, such as glycoproteins.

\section{Declarations}

\section{Acknowledgements}

The authors would like to acknowledge the support fromthe National Natural Science Foundation,Technology Department and Education Departmentof JiangxiProvince, and Nanchang University.

\section{Authors' contributions}

Huangand Y. Xiong conceived and designed the experiments. J. Hu, L. Ding, J. Chen, and K. Zhang performed the experiments. J. Hu, L. Ding, and Q. Guo conducted the data analysis.J. Hu and X. Huang wrote and revised the manuscript. L. Ding, X. Huang, and Y. Xiongsupported and supervised the research. All authors read and approved the final manuscript.

\section{Funding}

The study was supported by the National Natural Science Foundation, China (No.32160599, 32001788), the Jiangxi Provincial Natural Science Foundation (20202ACB215004), and theScientific Research Foundation of Education Department of JiangxiProvince (GJJ200221).

\section{Availability of data and materials}

All data used to support the findings of this study are available from the corresponding author upon request 


\section{Ethics approval and consent to participate}

Not applicable.

\section{Consent for publication}

All authors agree to publication.

\section{Competing interests}

The authors declare no competing interests.

\section{References}

1. Gaggin HK, Januzzi Jr JL. Biomarkers and diagnostics in heart failure. Biochim Biophys Acta, Mol Basis Dis. 2013; 1832:2442-2450.

2. Oremus M, McKelvie R, Don-Wauchope A, Santaguida PL, Ali U, Balion C, Hill S, Booth R, Brown JA, Bustamam A. A systematic review of BNP and NT-proBNP in the management of heart failure: overview and methods. Heart Failure Rev. 2014; 19:413-419.

3. Gong Y, Hu J, Choi JR, You M, Zheng Y, Xu B, Wen T, Xu F. Improved LFIAs for highly sensitive detection of BNP at point-of-care. Int J Nanomed. 2017; 12:4455.

4. Pu Q, Yang X, Guo Y, Dai T, Yang T, Ou X, Li J, Sheng S, Xie G. Simultaneous colorimetric determination of acute myocardial infarction biomarkers by integrating self-assembled 3D gold nanovesicles into a multiple immunosorbent assay. Microchim Acta. 2019; 186:138.

5. Wu J, Dong M, Santos S, Rigatto C, Liu Y, Lin F. Lab-on-a-chip platforms for detection of cardiovascular disease and cancer biomarkers. Sensors. 2017; 17:2934.

6. Zhuo Y, Yi W-J, Lian W-B, Yuan R, Chai Y-Q, Chen A, Hu C-M. Ultrasensitive electrochemical strategy for NT-proBNP detection with gold nanochains and horseradish peroxidase complex amplification. Biosens Bioelectron. 2011; 26:2188-2193.

7. Sinha A, Tai T-Y, Li K-H, Gopinathan P, Chung Y-D, Sarangadharan I, Ma H-P, Huang P-C, Shiesh S-C, Wang Y-L. An integrated microfluidic system with field-effect-transistor sensor arrays for detecting multiple cardiovascular biomarkers from clinical samples. Biosens Bioelectron. 2019; 129:155-163.

8. Fan D, Bao C, Liu X, Wu D, Zhang Y, Wang H, Du B, Wei Q. A novel label-free photoelectrochemical immunosensor based on NCQDs and $\mathrm{Bi}_{2} \mathrm{~S}_{3}$ co-sensitized hierarchical mesoporous $\mathrm{SnO}_{2}$ microflowers for detection of NT-proBNP.J Mater Chem B. 2018; 6:7634-7642.

9. Shi L, Li X, Zhu W, Wang Y, Du B, Cao W, Wei Q, Pang X. Sandwich-type electrochemiluminescence sensor for detection of NT-proBNP by using high efficiency quench strategy of $\mathrm{Fe}_{3} \mathrm{O}_{4} @ P D A$ toward $\mathrm{Ru}(\mathrm{bpy})_{3}{ }^{2+}$ coordinated with silver oxalate. ACS Sens. 2017; 2:1774-1778.

10. Alawieh $\mathrm{H}$, Chemaly TE, Alam S, Khraiche M. Towards point-of-care heart failure diagnostic platforms: BNP and NT-proBNP biosensors. Sensors (Basel). 2019; 19:5003. 
11. Kim HY, Park KS, Park HG. Glucose oxidase-like activity of cerium oxide nanoparticles: use for personal glucose meter-based label-free target DNA detection. Theranostics. 2020; 10:4507.

12. Wu W, Wang X, Shen M, Li L, Yin Y, Shen L, Wang W, Cui D, Ni J, Chen X. AlEgens barcodes combined with AlEgens nanobeads for high-sensitivity multiplexed detection. Theranostics. 2019; 9:7210.

13. Jans H, Liu X, Austin L, Maes G, Huo Q. Dynamic light scattering as a powerful tool for gold nanoparticle bioconjugation and biomolecular binding studies. Anal Chem. 2009; 81:9425-9432.

14. Levin AD, Ringaci A, Alenichev MK, Drozhzhennikova EB, Shevchenko KG, Cherkasov VR, Nikitin MP, Nikitin PI. Dynamic light scattering biosensing based on analyte-induced inhibition of nanoparticle aggregation. Anal Bioanal Chem. 2020; 412:3423-3431.

15. Xiong C, Ling L. Label-free, sensitive detection of $\mathrm{Hg}(\mathrm{II})$ with gold nanoparticles by using dynamic light scattering technique. Talanta. 2012; 89:317-321.

16. Huang X, Xu Z, Mao Y, Ji Y, Xu H, Xiong Y, Li Y. Gold nanoparticle-based dynamic light scattering immunoassay for ultrasensitive detection of Listeria monocytogenes in lettuces. Biosens Bioelectron. 2015; 66:184-190.

17. Wang X, Li Y, Quan D, Wang J, Zhang Y, Du J, Peng J, Fu Q, Zhou Y, Jia S, et al. Detection of hepatitis $B$ surface antigen by target-induced aggregation monitored by dynamic light scattering. Anal Biochem. 2012; 428:119-125.

18. Wang G, Yu M, Wang G. A versatile dynamic light scattering strategy for the sensitive detection of microRNAs based on plasmonic core-satellites nanoassembly coupled with strand displacement reaction. Biosens Bioelectron. 2019; 138:111319.

19. Gao Y, Xu S, He T, Li J, Liu L, Zhang Y, Ge S, Yan M, Liu H, Yu J. Ultrasensitive and specific microRNA detection via dynamic light scattering of DNA network based on rolling circle amplification. Sens Actuators B. 2020; 324.

20. Tavakkoli Yaraki M, Tan YN. Recent advances in metallic nanobiosensors development: colorimetric, dynamic light scattering and fluorescence detection. Sens Int. 2020; 1:100049.

21. Chen J, Hao L, Wu Y, Lin T, Li X, Leng Y, Huang X, Xiong Y. Integrated magneto-fluorescence nanobeads for ultrasensitive glycoprotein detection using antibody coupled boronate-affinity recognition. Chem Commun. 2019; 55:10312-10315.

22. Yan P, Ding Z, Li X, Dong Y, Fu T, Wu Y. Colorimetric sensor array based on Wulff-type boronate functionalized AgNPs at various pH for bacteria identification. Anal.Chem. 2019; 91:12134-12137.

23. Ren X-H, Wang H-Y, Li S, He X-W, Li W-Y, Zhang Y-K. Preparation of glycan-oriented imprinted polymer coating Gd-doped silicon nanoparticles for targeting cancer Tn antigens and dual-modal cell imaging via boronate-affinity surface imprinting. Talanta. 2021;223:121706.

24. Wang L, Yang J, Yang X, Hou Q, Liu S, Zheng W, Long Y, Jiang X. Mercaptophenylboronic acidactivated gold nanoparticles as nanoantibiotics against multidrug-resistant bacteria. ACS Appl Mater Interfaces. 2020; 12:51148-51159.

25. Li D, Chen Y, Liu Z. Boronate affinity materials for separation and molecular recognition: structure, properties and applications. Chem Soc Rev. 2015; 44:8097-8123. 
26. Schellenberger U, O'Rear J, Guzzetta A, Jue RA, Protter AA, Pollitt NS. The precursor to B-type natriuretic peptide is an O-linked glycoprotein. Arch Biochem Biophys. 2006; 451:160-166.

27. Hu B, Lu Y, Cui K, Yan Y. Molecular imprinting polymers based on boric acid-modified CdTe QDs for sensitive detection of glucose. Nano. 2018; 13:1850046.

28. Wang M, Xie J-L, Li J, Fan Y-Y, Deng X, Duan H-L, Zhang Z-Q. 3-Aminophenyl boronic acid functionalized quantum-dot-based ratiometric fluorescence sensor for the highly sensitive detection of tyrosinase activity. ACS Sens. 2020; 5:1634-1640.

29. Wilkins MD, Turner BL, Rivera KR, Menegatti S, Daniele M. Quantum dot enabled lateral flow immunoassay for detection of cardiac biomarker NT-proBNP. Sensing and bio-sensing research. 2018; 21:46-53.

30. Colom G, Salvador J-P, Acosta G, Albericio F, Royo M, Marco M-P. Competitive ELISA for N-terminal pro-brain natriuretic peptide (NT-proBNP) determination in human plasma. Analyst. 2020; 145:67196727.

31. Liang W, Li Y, Zhang B, Zhang Z, Chen A, Qi D, Yi W, Hu C. A novel microfluidic immunoassay system based on electrochemical immunosensors: An application for the detection of NT-proBNP in whole blood. Biosens Bioelectron. 2012; 31:480-485.

32. Feng J, Li F, Li X, Wang Y, Fan D, Du B, Li Y, Wei Q. Label-free photoelectrochemical immunosensor for NT-proBNP detection based on La-CdS/3D ZnIn ${ }_{2} \mathrm{~S}_{4} / \mathrm{Au} @ Z n O$ sensitization structure. Biosens Bioelectron. 2018; 117:773-780.

\section{Tables}

Table 1Comparison of analytical performances of the proposed method with other reported analytical technologies in detecting NT-proBNP.

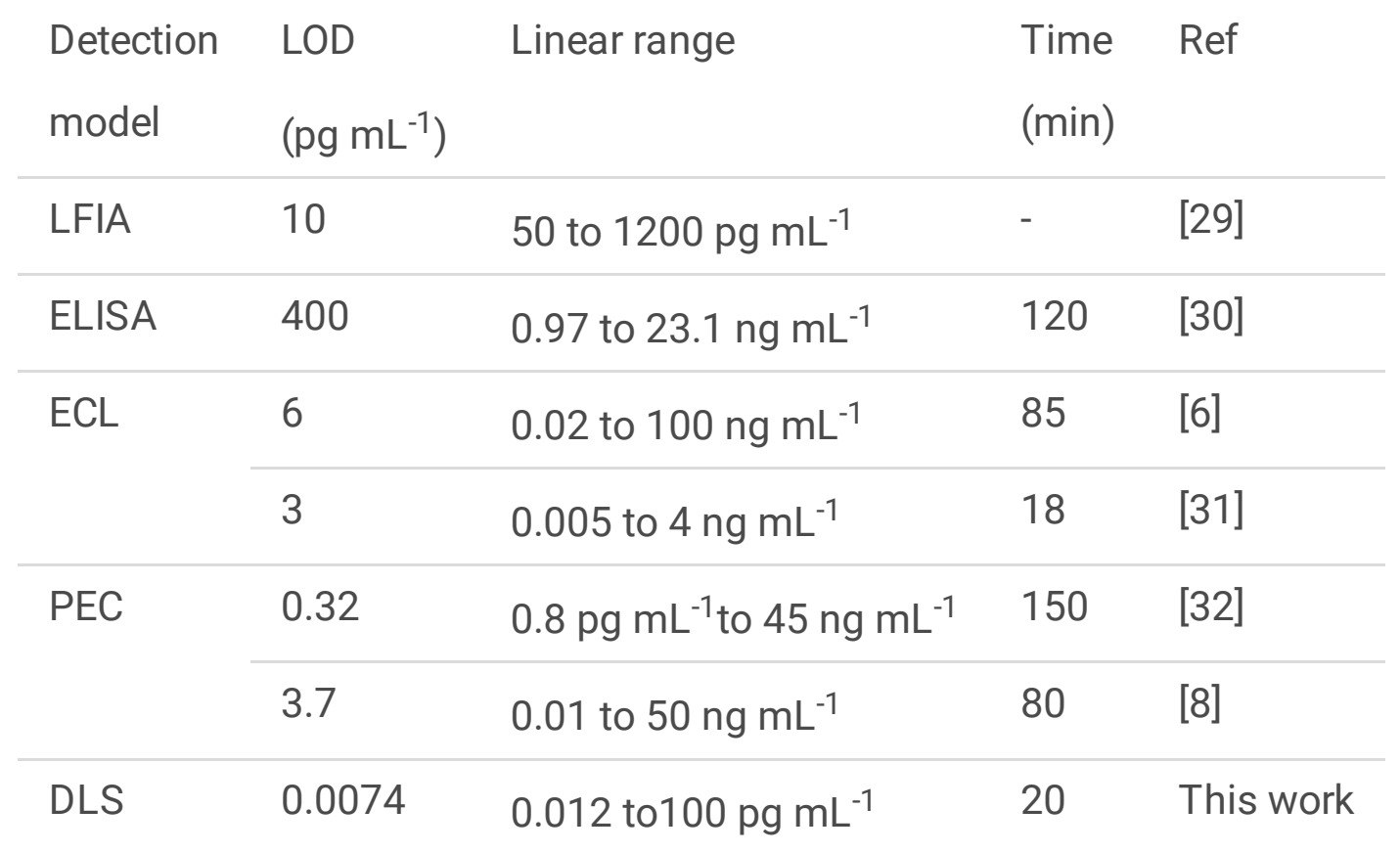


Note: DLS, dynamic light scattering;LFIA, Lateral flow immunoassay;ELISA,Enzyme linked immunosorbent assay;ECL, electrochemiluminesce; PEC, photoelectrochemical.

\section{Figures}
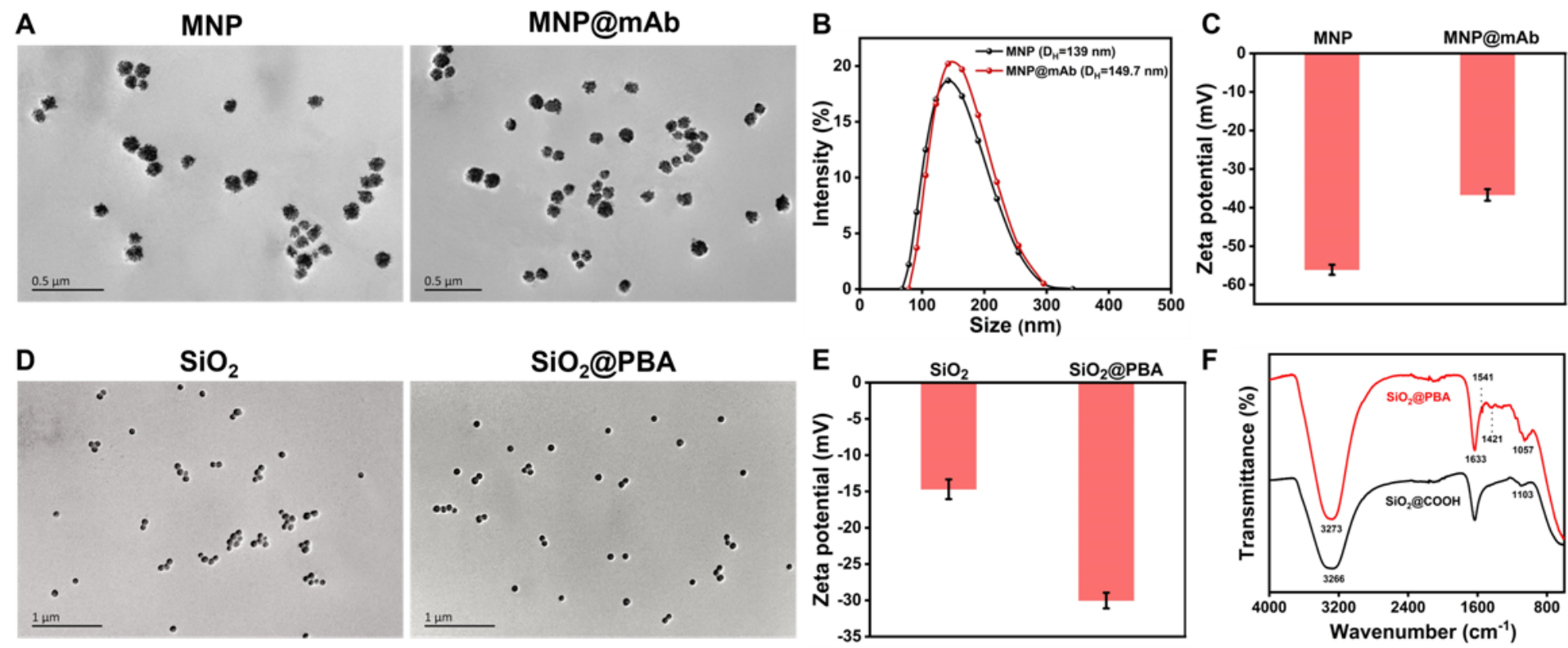

Figure 1

Characterization of the prepared MNP@mAb and SiO2@PBA conjugates. (A) TEM images of MNP and MNP@mAb. (B) The hydrodynamic diameter distribution of MNP and MNP@mAb. (C) The zeta potential of MNP and MNP@mAb. (D) TEM images of SiO2 and SiO2@PBA. (E) The zeta potential of SiO2 and SiO2@PBA. (F) The FTIR spectra of SiO2 (black curve) and SiO2@PBA (red curve). 
A

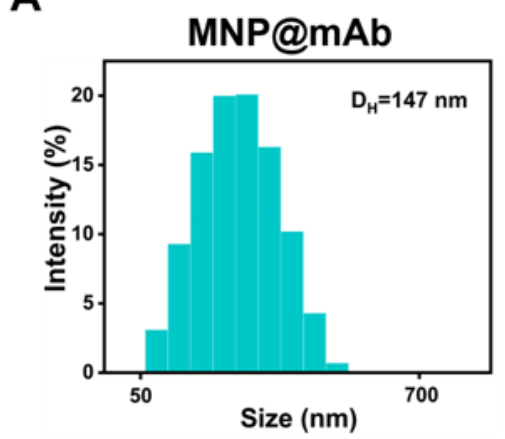

B

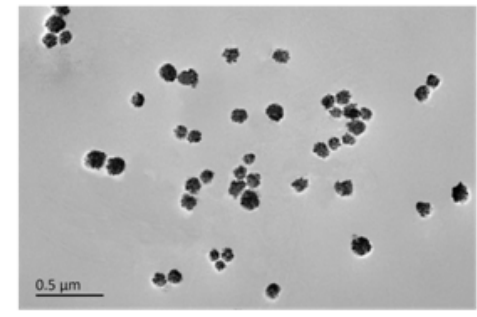

C

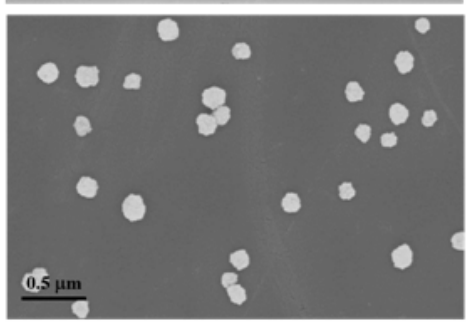

D

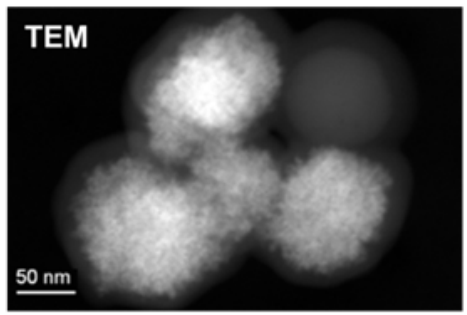

MNP@mAb+NT-proBNP
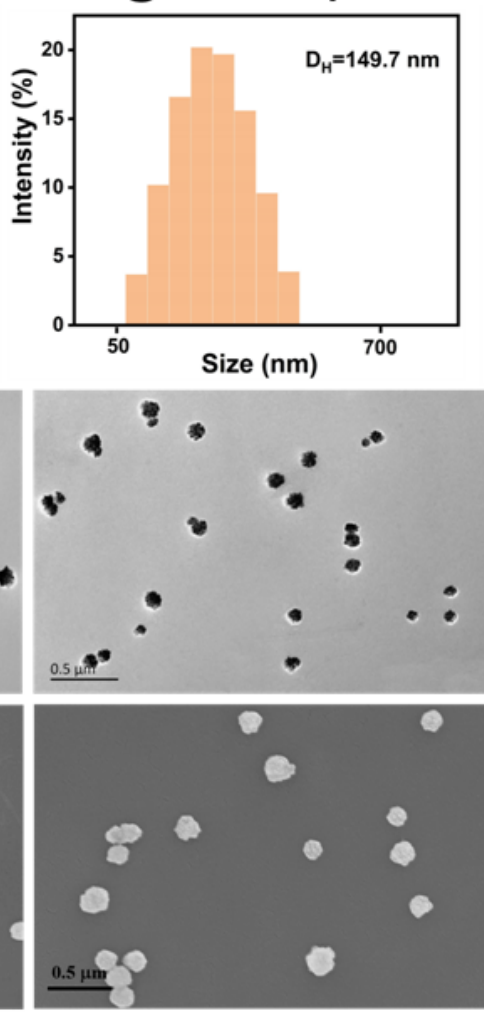

$\mathrm{Fe}$

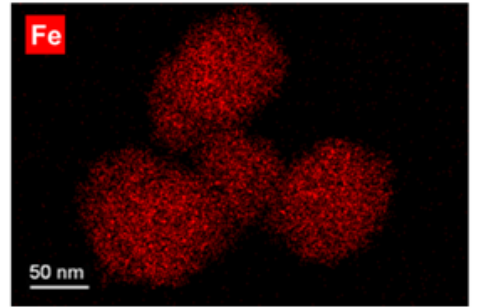

MNP@mAb+SiO
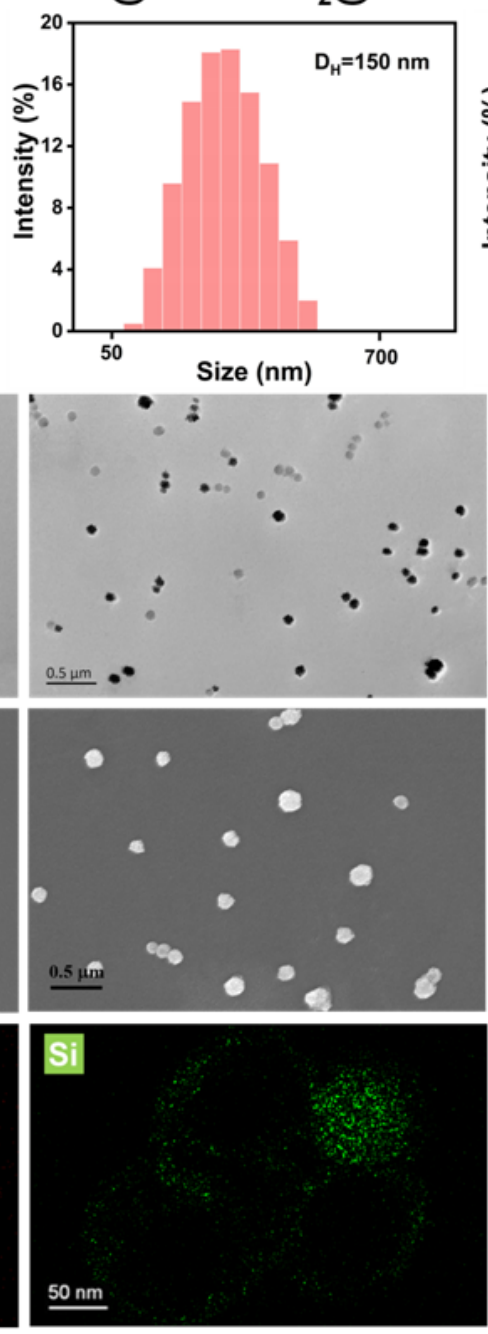

MNP@mAb+NT-proBNP

$+\mathrm{SiO}_{2} @ \mathrm{PBA}$
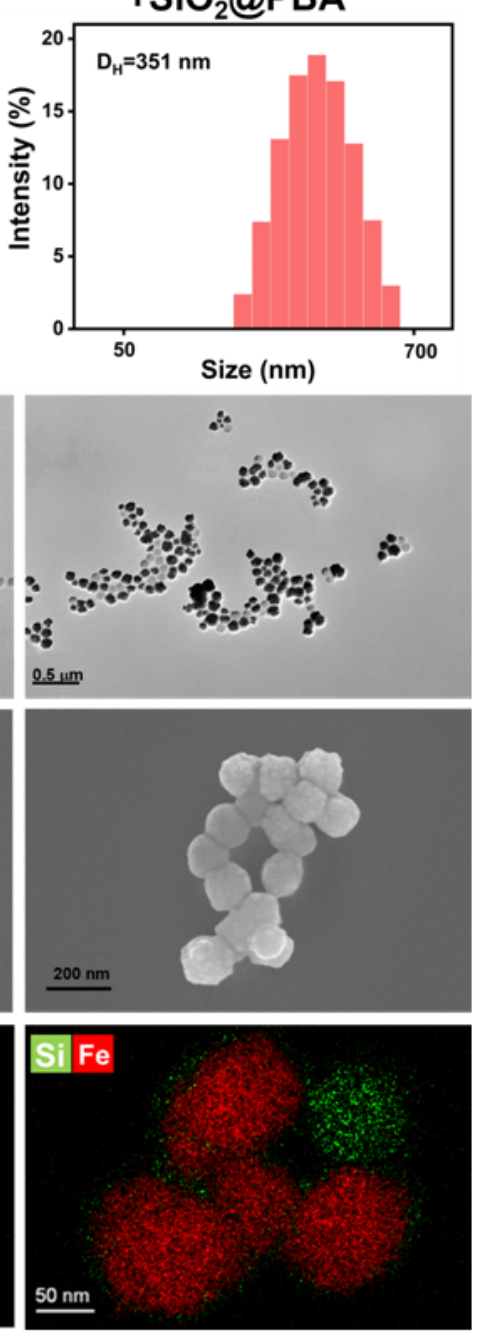

Figure 2

Verification of the developed DLS immunosensor for NT-proBNP detection by boronate affinity amplification. (A) Hydrodynamic diameter distribution, (B) TEM images, and (C) SEM images of MNP@mAb, MNP@mAb + NT-proBNP, MNP@mAb + SiO2@PBA, MNP@mAb + NT-proBNP + SiO2@PBA. (D) EDS mapping analysis of MNP@mAb + NT-proBNP + SiO2@PBA. 

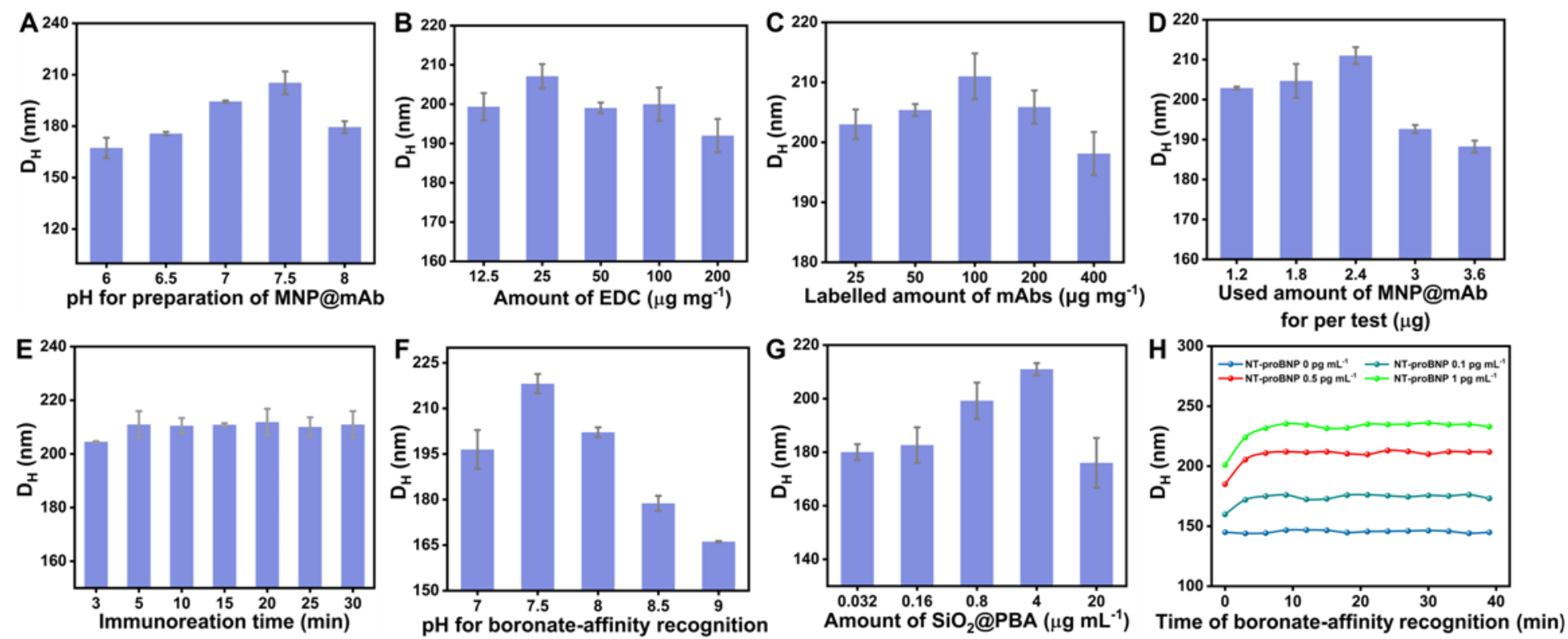

Figure 3

Parameter optimization. (A) pH value. (B) EDC amount. (C) The saturated labelling amount of mAbs for the preparation of MNP@mAb. (D) The used amount of MNP@mAb for per test. (E) The immunoreaction time of MNP@mAb for the capture of BNP from the sample solution. $(F)$ The solution pH of boronate affinity recognition between NT-proBNP and Si02@PBA. (G) The used amount of SiO2@PBA. (H) The reaction time of boronate affinity recognition between NT-proBNP and SiO2@PBA. 

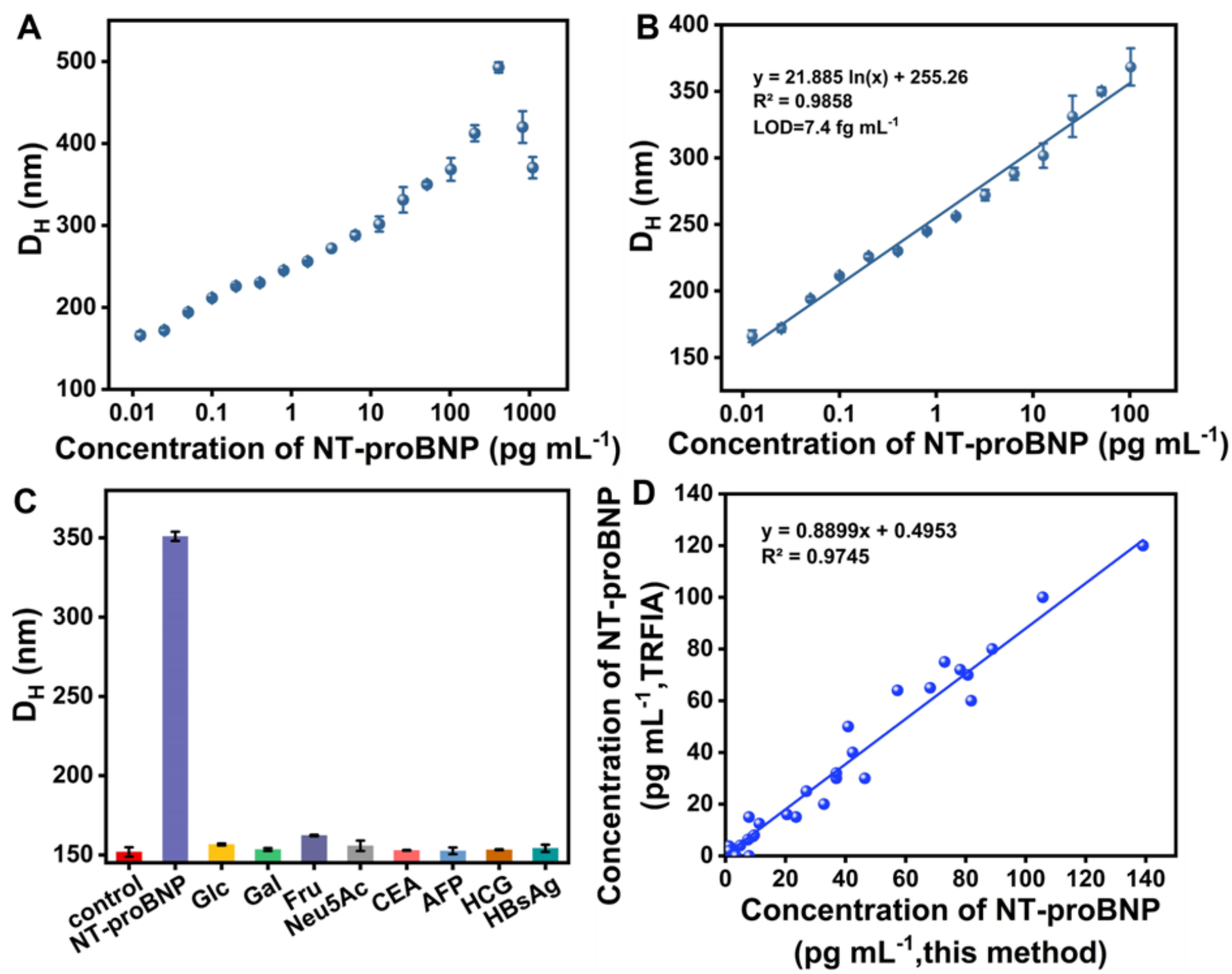

Figure 4

(A) The dose-response relationship between the DH and the NT-proBNP concentration ranged from 0.012 to $1100 \mathrm{pg} \mathrm{mL}-1$. (B) Standard curve for NT-proBNP detection with the concentration ranged from 0.012 to $100 \mathrm{pg} \mathrm{mL-1}$. (C) Specificity evaluation for NT-proBNP (20 pg mL-1) by analyzing other common nontarget proteins and saccharides, including Glc (5 mg mL-1), Gal (5 mg mL-1), Fru (5 mg mL-1), Neu5Ac (1 $\mathrm{mg} \mathrm{mL}-1)$, CEA (1 ng mL-1), AFP (1 ng mL-1), HCG (500 mlU mL-1), and HBsAg (1 ng mL-1). (D) A correlation analysis between the detection results obtained from the developed DLS immunosensor and the TRFIA in detecting 30 NT-proBNP-positive serum samples.

\section{Supplementary Files}

This is a list of supplementary files associated with this preprint. Click to download.

- Graphicabstract.tif 
- Scheme1.png

- SIHuetal20211014.docx 